OPEN ACCESS

Edited by:

Atsushi Otsuka,

Kyoto University, Japan

Reviewed by:

Teruki Yanagi,

Hokkaido University Hospital, Japan

Alessandro Poggi,

Ospedale Policlinico

San Martino, Italy

Carlos Alfaro,

Universidad de Navarra, Spain

${ }^{*}$ Correspondence:

Taku Fujimura

tfujimura1@mac.com

Specialty section: This article was submitted to

Cancer Immunity and Immunotherapy,

a section of the journal

Frontiers in Oncology

Received: 29 November 2017 Accepted: 05 January 2018 Published: 23 January 2018

Citation:

Fujimura T, Kambayashi Y, Fujisawa Y, Hidaka T and Aiba S (2018)

Tumor-Associated Macrophages:

Therapeutic Targets for Skin Cancer.

Front. Oncol. 8:3.

doi: 10.3389/fonc.2018.00003

\section{Tumor-Associated Macrophages: Therapeutic Targets for Skin Cancer}

\author{
Taku Fujimura ${ }^{1 *}$, Yumi Kambayashi', Yasuhiro Fujisawa ${ }^{2}$, Takanori Hidaka' \\ and Setsuya Aiba'
} 'Department of Dermatology, Tohoku University Graduate School of Medicine, Sendai, Japan, ${ }^{2}$ Department of Dermatology,
University of Tsukuba, Tsukuba, Japan

Tumor-associated macrophages (TAMs) and regulatory $\mathrm{T}$ cells (Tregs) are significant components of the microenvironment of solid tumors in the majority of cancers. TAMs sequentially develop from monocytes into functional macrophages. In each differentiation stage, TAMs obtain various immunosuppressive functions to maintain the tumor microenvironment (e.g., expression of immune checkpoint molecules, production of Treg-related chemokines and cytokines, production of arginase I). Although the main population of TAMs is immunosuppressive M2 macrophages, TAMs can be modulated into M1-type macrophages in each differential stage, leading to the suppression of tumor growth. Because the administration of certain drugs or stromal factors can stimulate TAMs to produce specific chemokines, leading to the recruitment of various tumorinfiltrating lymphocytes, TAMs can serve as targets for cancer immunotherapy. In this review, we discuss the differentiation, activation, and immunosuppressive function of TAMs, as well as their benefits in cancer immunotherapy.

\section{Keywords: tumor-associated macrophages, immunosuppression, M2 polarization, chemokines, angiogenetic factors, regulatory $\mathrm{T}$ cells}

\section{INTRODUCTION}

Tumor-associated macrophages (TAMs) and regulatory T cells (Tregs) are significant components of the tumor microenvironment $(1,2)$. TAMs express immune checkpoint modulators [e.g., B7 family, B7-homolog family including programmed death ligand 1 (PD-L1)] (3) that directly suppress activated $\mathrm{T}$ cells. In addition, TAMs produce various chemokines that attract other immunosuppressive cells such as Tregs, myeloid-derived suppressor cells (MDSCs), and type 2 helper (Th2) $\mathrm{T}$ cells, which maintain the immunosuppressive factors of the tumor microenvironment $(1,2,4)$. Moreover, TAMs also produce matrix metalloproteinases (MMPs), which play critical roles in tissue remodeling associated with various physiological processes such as morphogenesis, angiogenesis, tissue repair, local invasion, and metastasis $(1,5,6)$. TAMs have been detected in various skin cancers such as melanoma, squamous cell carcinoma (SCC), extramammary Paget's disease (EMPD), Merkel cell carcinoma, basal cell carcinoma, and mycosis fungoides (MFs) (1,2,7-15) (Table 1). Because the stromal factor on each cancer stem cell is an important factor for TAM stimulation, leading to the induction of specific TAM phenotypes, investigating the immunomodulatory stromal cells in the tumor microenvironment is important for establishing the appropriate immunotherapy for each type of cancer $(1,8,9,16,17)$. In addition, it may be possible to repolarize TAMs into anti-tumor macrophages, such as M1-phenotype macrophages, to suppress tumor progression by modifying the profiles of tumor-infiltrating lymphocytes (TILs) $(7,18,19)$. Thus, TAMs could be a target for immunotherapy in skin cancers $(1,2)$. In this review, we discuss the differentiation, activation, and 
TABLE 1 | Tumor-associated macrophages in skin cancer: mouse and human models.

\begin{tabular}{|c|c|c|c|c|c|}
\hline Cancer species & Mouse (reference) & Human (reference) & Depletion & Reprogrammed & Biomarkers \\
\hline Malignant melanoma & $(3,7,13,19,20,22,39,51,62,63,64,65)$ & $(7,35,59,60)$ & $(13,65)$ & $(5,19,20,22,35,39)$ & $(3,59,60,61)$ \\
\hline Cutaneous squamous cell carcinoma & $(23,24,32)$ & $(11,12,34)$ & (23) & $(24,32)$ & $(11,12)$ \\
\hline Merkel cell carcinoma & - & $(14,36)$ & & & $(14,36)$ \\
\hline Extramammary Paget's disease & - & $(8,17)$ & & $(17)$ & (8) \\
\hline Basal cell carcinoma & (26) & $(15)$ & $(26)$ & & $(15)$ \\
\hline Dermatofibrosarcoma protuberans & - & (5) & & & (5) \\
\hline Cutaneous T cell lymphoma & $(25)$ & $(9,18,28,29,30,31,57)$ & $(25)$ & $(18,57)$ & $(9,28,29,30)$ \\
\hline
\end{tabular}

immunosuppressive function of TAMs, as well as their benefit in cancer immunotherapy.

\section{DIFFERENTIATION AND ACTIVATION OF TAMs IN TUMORS}

Tumor-associated macrophages are characterized by their heterogeneity and plasticity, as they can be functionally reprogrammed to polarized phenotypes by exposure to cancer-related factors, stromal factors, infections, or even drug interventions $(1,2,7,9,11,17,19)$. Because TAMs sequentially differentiate from monocytes into functional macrophages through multiple steps, they have heterogeneity and plasticity in cancer (Figure 1). Monocytes recruited from the circulation differentiate into tissue macrophages by macrophage colony-stimulating factor (M-CSF), and are primed with several cytokines such as interferon gamma (IFN- $\gamma$ ), interleukin 4 (IL-4), and IL-13 (2). Thereafter, macrophages change their functional phenotype in response to environmental factors or even tumor-derived protein stimulation $(2,8,17)$. In skin cancer, for example, targeting the M-CSF receptor with anti-CSF short interfering RNA (siCD115) in TAMs led to modulation of the TIL profile, resulting in growth suppression of B16 melanoma in vivo (20). In the second phase of priming, type I IFN (IFN- $\alpha$, IFN- $\beta$ ) and type II IFN (IFN- $\gamma$ ) modulate the production of chemokines from TAMs, suggesting that these cytokines repolarize TAMs in several skin cancers $(7,18)$. Cancer stromal factors such as soluble receptor activator of nuclear factor kappa-B ligand (RANKL) derived from cancer cells could be a third mode of stimulation that activates mature M2 macrophages to produce a series of chemokines that recruit immunosuppressive cells such as Tregs and Th2, leading to maintenance of the tumor microenvironment $(8,10,17)$. These reports suggest that each of these three differentiation steps could serve as a target for immunotherapies.

\section{ROLES OF TAMS IN MAINTAINING THE IMMUNOSUPPRESSIVE MICROENVIRONMENT}

\section{Chemokines from TAMs Determine the Immunological Microenvironment in Tumors}

Chemokines play crucial roles in determining the profiles of TILs in the tumor microenvironment, and the profiles of chemokines

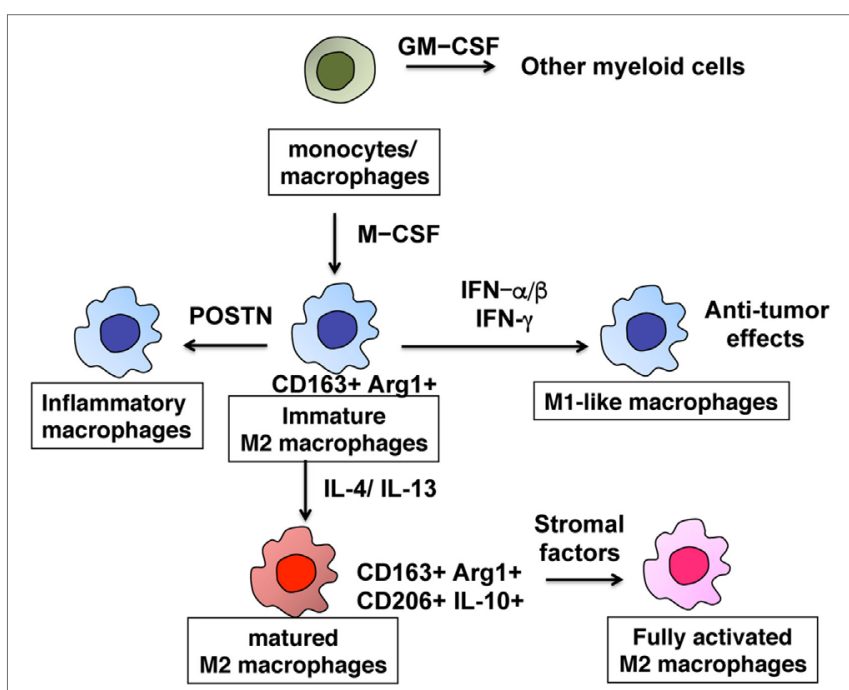

FIGURE 1 | Differentiation of M2-polarized tumor-associated macrophages. The multiple steps of the development of monocytes into fully functional macrophages.

from TAMs are determined by stromal factors of each skin cancer (1). For example, immune cells in the tumor microenvironment determine the aggressiveness of melanoma (21). In metastatic melanoma, periostin (POSTN) is expressed in the region surrounding melanoma cell nests in metastatic melanoma lesions that develop at the wound site (16). In addition, TAMs are prominent in the tumor stroma in melanoma $(7,19,22)$, and POSTN stimulates $\mathrm{CD} 63^{+}$macrophages to produce several specific cytokines including Treg-related chemokines [chemokine ligand 17 (CCL17), CCL22] (9). Because CCL17 and CCL22 from TAMs attracts Tregs to the tumor site in melanoma $(7,21,22)$, repolarization of TAMs by immunomodulatory reagents such as IFN- $\beta$ and imiquimod are useful for suppressing tumor growth in melanoma $(7,22)$. The downregulation of CCL22 production was also observed in B16F10 melanoma mouse treated with classical cytotoxic anti-melanoma drugs such as dacarbazine, nimustine hydrochloride, and vincristine, all of which have been used in the adjuvant setting for advanced melanoma for the last 30 years (19). Other reports have suggested that a series of chemokines (CCL17, CXCL10, CCL4, and IL-8) in cerebrospinal fluid may be useful for predicting brain metastasis in melanoma patients (21). Together, these reports suggest the significance of chemokines from TAMs that can be induced by POSTN in the 
tumor stroma to induce melanoma-specific TILs in patients with melanoma.

Tumor-associated macrophages in non-melanoma skin cancer also secrete an array of chemokines in lesional skin to regulate the tumor microenvironment (1). In EMPD, for example, soluble RANKL released by Paget cells increases the production of CCL5, CCL17, and CXCL10 from RANK ${ }^{+}$M2 polarized TAMs $(8,10,17)$, suggesting that Paget cells can determine the immunological microenvironment by the stimulation of TAMs. The results of this study led to the hypothesis that denosumab, a full human monoclonal antibody for RANKL, has therapeutic effects in invasive EMPD. In cutaneous squamous cell carcinoma (cSCC), according to its heterogeneity of differentiation of cancer cells, TAMs in cSCC heterogeneously polarized from M1 to M2 (11). Indeed, Petterson et al. (11) reported that CD163 ${ }^{+}$TAMs not only express CCL18 (11), an M2 chemokine involved in remodeling of the tumor microenvironment but are also colocalized with phosphorylated signal transducer and activator of transcription 1 (11), suggesting the heterogeneous activation states of TAMs. Although the exact stimulator of cSCC is unknown, the depletion of TAMs such as antibody-mediated depletion (e.g., anti-CSF1R Ab) or bisphosphonate could be a useful therapy for unresectable cSCC (23-26).

Not only solid tumors but also hematopoietic malignancies in the skin contain CD163+ TAMs $(25,27-29)$, which produce chemokines that direct to specific anatomic sites to form metastases (25). Indeed recently, Wu et al. (9) used a human xenograft CTCL cell model to demonstrate that chemokines from TAMs play crucial roles in tumor formation in MF lesions. In another report, it was shown that the cancer stroma of MF containing POSTN and IL-4 might stimulate TAMs to produce chemokines that correlate with tumor formation in MF (25), and that chemokines from TAMs can be modified by immunomodulatory agents such as IFN- $\alpha$ and IFN- $\gamma$, leading to their therapeutic effects (18). Furthermore, CCL18 produced by TAMs in MF at the invasive margin of the tumor promote the recruitment of CTCL cells, leading to cancer progression (30). These reports suggest the significance of chemokines from TAMs for the development of CTCL.

\section{Direct Suppressive Function of TAMs}

Immunomodulatory costimulatory molecules, such as B7 homologs, play representative roles in the direct cell-mediated suppressive mechanism of TAMs. Recently, several reports have suggested that the expression of PD-L1 (also known as B7H1) in TAMs is necessary for antigen-specific tolerance induction $(1,3,31)$ in tumor-bearing hosts. For example, the expression of PD-L1 on TAMs is augmented by autocrine IL-10 from M2-polarized TAMs stimulated by specific antigens (31). Another report showed that the decrease of IL-10 in MDSCs led to the downregulation of PD-L1 expression in MDSC in a mouse melanoma model (3). Linde et al. (32) reported that IL-10polarized TAMs into M2 phenotypes in the presence of IL-4 and vascular endothelial growth factor A (VEGF-A) in cSCC. These reports suggest that IL-10 upregulates PD-L1 expression on TAMs, inducing immunosuppression in the tumor microenvironment in the skin. Arginase 1 is one of the key factors for the suppressive function of TAMs. Its expression is widely detected in immature and functional M2 macrophages $(1,8,17)$, leading to suppression of T cell activity by L-arginine catabolism (33). Indeed, $\mathrm{CD}_{163^{+}}$TAMs expresses arginase 1 in several skin cancers such as EMPD and SCC $(8,34)$. More recently, Pico de Coaña et al. (35) reported the additional immunomodulatory effects of ipilimumab on granulocytic MDSCs, which are circulating macrophages in tumor-bearing hosts, suggesting the crosstalk between Tregs and granulocytic MDSCs through the CTLA4/B7 homolog pathway and the significance of the direct suppressive function of TAMs (35).

\section{Angiogenetic Factors from TAMs}

Tumor-associated macrophages produce angiogenetic factors such as VEGF, platelet-derived growth factor, and transforming growth factor $\beta$, or by expressing MMPs to induce neovascularization $(10,28,32,36-38)$. Linde et al. (32) reported that VEGF-A augments the recruitment of TAMs at a tumor site by promoting neovascularization in a mouse skin tumor model (32). In a human skin cancer model, Werchau et al. (36) reported that VEGF-C expressed by TAMs contributes to lymphangiogenesis and the progression of Merkel cell carcinoma (36). In angiosarcoma, TAMs express MMP9, which might be a target for amino bisphosphonate (37). Another report suggested that inhibition of the VEGF/VEGF receptor pathway inhibits M2 polarization in TAMs, leading to reduced vascular density and tumor growth in MCA205 mouse sarcoma (38). In addition, more recently, Yamada et al. (39) reported that the expression of MGF-E8 on mesenchymal stromal cells plays crucial roles in inducing M2 macrophage polarization, leading to suppression of tumor growth by the reduction of VEGF expression in TAMs in B16F10 melanoma. These reports indicate the significance of VEGF produced by M2 macrophages in tumor progression, and show that both VEGF and MMPs are key markers for M2 macrophages in skin cancers $(11,40,41)$. For example, in a melanoma model, osteopontin signaling promoted macrophage recruitment by the secretion of prostaglandin E2 and MMP-9 from TAMs, leading to angiogenesis and tumor progression (41). These reports suggest that MMPs play crucial roles in tumor progression. MMPs can also be produced by TAMs upon stimulation of stromal proteins in skin cancer $(9,10)$. For example, the stimulation of POSTN augments the production of MMP1 and MMP12 from monocyte-derived immature M2 macrophages (9). Because POSTN is abundant in the tumor stroma of MF and dermatofibrosarcoma protuberans (DFSP) $(5,9)$, and because substantial numbers of CD163+ TAMs have been detected in the POSTN-rich area in the lesional skin of skin tumors $(5,9)$, the production of MMP1 and MMP12 is prominent in the lesional skin of MF and DFSP. Notably, as reported by Livtinov et al. (42), among the MMPs, only MMP12 is a risk factor for CTCL progression, as determined by transcriptional profiling (42). RANKL is expressed in skin cancers of apocrine origin such as EMPD and apocrine carcinoma $(8,37)$, and is released in its soluble form. Because monocyte-derived M2 macrophages produce MMP1 and MMP25 by RANKL stimulation, TAMs in skin cancer of apocrine origin produce MMP1 and MMP25 at the tumor site (37). These reports suggest that TAMs stimulated by tumor stromal 
factors play roles in the carcinogenesis of these skin cancers, and might be targets for molecular-targeted therapy in the future.

\section{CLINICAL BENEFITS OF TAMS}

\section{The Effects of Anticancer Drug for TAMs}

Because TAMs comprise the immunosuppressive microenvironment at the tumor site, they may be optimal therapeutic targets in cancer (1, 2, 4, 43-46). For example, Rogers et al. (44) reported the immunomodulatory effects of bisphosphonate on TAMs in patients with breast and prostate cancers upon the repolarization of TAMs into tumoricidal macrophages (44). More recently, several reports have also focused on the immunomodulatory effects of chemotherapeutic reagents on TAMs $(19,47,48)$. For example, a non-cytotoxic dose of paclitaxel decreased MDSCs and even blocked the immunosuppressive potential of MDSCs in a mouse melanoma model (47). More recently, Fujimura et al. (19) reported the immunomodulatory effects of cytotoxic anti-melanoma drugs, dacarbazine, nimustine hydrochloride, and vincristine, on TAMs both in vitro and in vivo by inhibition of STAT3 signals (19). The authors concluded that their immunomodulatory effects could explain their antitumor effects in postoperative melanoma patients. Peplomycin administered through a superficial temporal artery using an intravascular indwelling catheter, which can cause dose-independent interstitial pneumonia (49), decreased the number of TAMs and Tregs in CSCC on the lips, leading to an increase in the number of immunoreactive cells at the tumor sites (50), and possible autoimmune-like interstitial pneumonia $(49,50)$. More recently, not only cytotoxic chemotherapeutic drugs but also low molecular weight compounds were reported to co-localize with TAMs at tumor sites. Indeed, Hu-Lieskovan et al. (13) reported that single-agent dabrafenib increased TAMs and Tregs in melanoma, which decreased with the addition of trametinib, leading to the synergistic effects of immune checkpoints inhibitors with dabrafenib and trametinib combination therapy. In another report, the anti-macrophage receptor with collagenous structure was reported to polarize TAMs into proinflammatory phenotypes to induce anti-melanoma immune response in B16 melanomas (51). In addition, Gordon et al. (52) reported that inhibition of PD-1/PD-L1 in vivo increased macrophage phagocytosis, reduced tumor growth, and prolonged the survival of macrophages. In another report, increasing expression levels of PD-L1 in TAMs, 2 months after the administration of anti-PD-1 Abs in patients with advanced melanoma, was correlated with the response to immunotherapy (53), suggesting that PD-L1 expression in TAMs could be a biomarker that predicts the effectiveness of anti-PD-1 Ab therapy. Because the anti-PD-1 Abs nivolumab and pembrolizumab are widely used to treat advanced cancer, including melanoma (53), one target of anti-PD-1 Abs in patients with advanced melanoma could be an immunomodulatory effect on TAM, which, in turn, might be correlated with both their effectiveness and the development of adverse events. TAMs produce not only chemokines that directly recruit immunosuppressive cells to the tumor microenvironment but also produce cytokines that stimulate other stromal cells such as fibroblasts to produce chemokines $(54,55)$. Indeed, Young et al. (54) reported that IL- $1 \beta$ from TAMs stimulate fibroblasts to produce CXCR2 ligand, which plays crucial roles in recruiting granulocytic MDSCs to tumor sites $(55,56)$. The authors concluded that CXCR2 agonists in combination with anti-CD115 Abs could suppress B16F10 melanoma in vivo by inhibiting the recruitment of granulocytic MDSCs and depletion of immature TAMs (56). Interestingly, the antihuman CD115 Ab, emactuzumab, decreased the number of $\mathrm{CD} 163^{+} \mathrm{CD}^{2} 06^{+} \mathrm{M} 2$ macrophages in patients with melanoma by depleting immature TAMs before the IL- 4 stimulation phase (57). Together, these reports suggest that anti-CXCR2 agonists in combination with emactuzumab might induce the antimelanoma immune response by reducing the number of M2 polarized TAMs. These reports suggest the significance of assessing the effects of chemotherapeutic drugs on TAMs $(13,19,47,49,50)$.

\section{TAMs as a Biomarker for Disease Activity and Adverse Events}

As described above, because TAMs produce tumor-specific chemokines by the stimulation of stromal factors, chemokines might serve as biomarkers that reflect disease activity. For example, TAMs produced CCL18 in the lesional skin of CTCL (26), which reflect disease severity and prognosis (58). Immunomodulatory reagents such as IFNs and imiquimod reduce CCL22 from TAMs, leading to the therapeutic effects of them in mouse B16F10 melanoma models $(7,22)$. CCL5, which induces Th2 cells from naive T cells (59), reflects the cancer stage and disease progression in gastric cancers (60). Another TAM-associated factor, sCD163, could be a useful biomarker for cancer treatment, as it is an activation marker for $\mathrm{CD} 163^{+}$tissue macrophages that is present in the serum as a result of proteolytic shedding (61). Serum sCD163 levels increase in autoimmune diseases such as atherosclerosis, rheumatoid arthritis, moyamoya disease, pemphigus vulgaris, and bullous pemphigoid (62-64), and reflect disease activity (61). Therefore, as we previously reported, sCD163 is a possible marker for predicting immune-related adverse events caused by immune checkpoints inhibitors $(64,65)$. These reports suggested that the production derived from TAMs could be a biomarker for cancer treatment in the future.

\section{CONCLUDING REMARKS}

Although several studies have suggested that high numbers of TAMs in tumor-bearing individuals are associated with a poor prognosis, making them useful as prognostic markers in cancer, further studies are needed to quantify their impact in different cancers.

\section{AUTHOR CONTRIBUTIONS}

FT designed the study. FT, KY, and HT wrote the article. FT, FY, and AS supervised the study.

\section{FUNDING}

This study was supported in part by a Grant-in-Aid for scientific research from the Japan Society for the Promotion of Science (No.: 16K10143). 


\section{REFERENCES}

1. Fujimura T, Kakizaki A, Furudate S, Kambayashi Y, Aiba S. Tumor-associated macrophages in skin: how to treat their heterogeneity and plasticity. J Dermatol Sci (2016) 83(3):167-73. doi:10.1016/j.jdermsci.2016.05.015

2. Noy R, Pollard JW. Tumor-associated macrophages: from mechanisms to therapy. Immunity (2014) 41:49-61. doi:10.1016/j.immuni.2014.06.010

3. Fujimura T, Ring S, Umansky V, Mahnke K, Enk AH. Regulatory T cells (Treg) stimulate B7-H1 expression in myeloid derived suppressor cells (MDSC) in ret melanomas. J Invest Dermatol (2012) 132(4):1239-46. doi:10.1038/jid. 2011.416

4. Fujimura T, Mahnke K, Enk AH. Myeloid derived suppressor cells and their role in tolerance induction in cancer. J Dermatol Sci (2010) 59(1):1-6. doi:10.1016/j.jdermsci.2010.05.001

5. Fujimura T, Kakizaki A, Sato Y, Tanita K, Furudate S, Aiba S. The immunological roles of periostin/tumor-associated macrophage axis in development of dermatofibrosarcoma protuberans. Anticancer Res (2017) 37(6): 2867-73. doi:10.21873/anticanres.11639

6. Baay M, Brouwer A, Pauwels P, Peeters M, Lardon F. Tumor cells and tumorassociated macrophages: secreted proteins as potential targets for therapy. Clin Dev Immunol (2011) 2011:565187. doi:10.1155/2011/565187

7. Kakizaki A, Fujimura T, Furudate S, Kambayashi Y, Yamauchi T, Yagita H, et al. Immunomodulatory effect of peritumoral administration of interferon-beta on melanoma through tumor-associated macrophages. Oncoimmunology (2015) 4(11):e1047584. doi:10.1080/2162402X.2015.1047584

8. Kambayashi Y, Fujimura T, Furudate S, Asano M, Kakizaki A, Aiba S. The possible interaction between receptor activator of nuclear factor kappa-B ligand (RANKL) expressed by extramammary Paget cells and its ligand on dermal macrophages. J Invest Dermatol (2015) 135(10):2547-50. doi:10.1038/ jid.2015.199

9. Furudate S, Fujimura T, Kakizaki A, Kambayashi Y, Asano M, Watabe A, et al. The possible interaction between periostin expressed by cancer stroma and tumor-associated macrophages in developing mycosis fungoides. Exp Dermatol (2016) 25(2):107-12. doi:10.1111/exd.12873

10. Fujimura T, Kambayashi Y, Furudate S, Kakizaki A, Hidaka T, Asano M, et al. Receptor activator of nuclear factor kappa-B ligand (RANKL)/RANK signaling promotes cancer-related inflammation through M2 macrophages. Exp Dermatol (2016) 25(5):397-9. doi:10.1111/exd.12949

11. Pettersen JS, Fuentes-Duculan J, Suárez-Fariñas M, Pierson KC, Pitts-Kiefer A, Fan L, et al. Tumor-associated macrophages in the cutaneous SCC microenvironment are heterogeneously activated. JInvest Dermatol (2011) 131(6):1322-30. doi:10.103/jid.2011.9

12. Kambayashi Y, Fujimura T, Aiba S. Comparison of immunosuppressive and immunomodulatory cells in keratoacanthoma and cutaneous squamous cell carcinoma. Acta Derm Venereol (2013) 93(6):663-8. doi:10.2340/ 00015555-1597

13. Hu-Lieskovan S, Mok S, Homet Moreno B, Tsoi J, Robert L, Goedert L, et al. Improved antitumor activity of immunotherapy with BRAF and MEK inhibitors in BRAF(V600E) melanoma. Sci Transl Med (2015) 7(279):ra41. doi:10.1126/scitranslmed.aaa4691

14. Fujimura T, Furudate S, Kambayashi Y, Kakizaki A, Yamamoto Y, Okuhira H, et al. Phospho-STAT5B expression is a prognostic marker for Merkel cell carcinoma. Anticancer Res (2017) 37(5):2335-41. doi:10.21873/anticanres. 11571

15. Tjiu JW, Chen JS, Shun CT, Lin SJ, Liao YH, Chu CY, et al. Tumor-associated macrophage-induced invasion and angiogenesis of human basal cell carcinoma cells by cyclooxygenase-2 induction. J Invest Dermatol (2009) 129(4):1016-25. doi:10.1038/jid.2008.310

16. Fukuda K, Sugihara E, Ohta S, Izuhara K, Funakoshi T, Amagai M, et al. Periostin is a key niche component for wound metastasis of melanoma. PLoS One (2015) 10(6):e0129704. doi:10.1371/journal.pone.0129704

17. Fujimura T, Kambayashi Y, Furudate S, Asano M, Kakizaki A, Aiba S. Receptor activator of nuclear factor kappa-B ligand (RANKL) promotes the production of CCL17 from RANK+ M2 macrophages. J Invest Dermatol (2015) 135(11):2884-7. doi:10.1038/jid.2015.209

18. Furudate S, Fujimura T, Kakizaki A, Hidaka T, Asano M, Aiba S. Tumorassociated M2 macrophages in mycosis fungoides acquire immunomodulatory function by interferon alpha and interferon gamma. J Dermatol Sci (2016) 83(3):182-9. doi:10.1016/j.jdermsci.2016.05.004
19. Fujimura T, Kakizaki A, Kambayashi Y, Sato Y, Tanita K, Lyu C, et al. Cytotoxic anti-melanoma drugs suppress the activation of M2 macrophages. Exp Dermatol (2018) 27(1):64-70. doi:10.1111/exd.13417

20. Qian Y, Qiao S, Dai Y, Xu G, Dai B, Lu L, et al. Molecular-targeted immunotherapeutic strategy for melanoma via dual-targeting nanoparticles delivering small interfering RNA to tumor-associated macrophages. ACS Nano (2017) 11(9):9536-49. doi:10.1021/acsnano.7b05465

21. Lok E, Chung AS, Swanson KD, Wong ET. Melanoma brain metastasis globally reconfigures chemokine and cytokine profiles in patient cerebrospinal fluid. Melanoma Res (2014) 24(2):120-30. doi:10.1097/CMR.0000000000000045

22. Furudate S, Fujimura T, Kambayashi Y, Kakizaki A, Hidaka T, Aiba S. Immunomodulatory effect of imiquimod through CCL22 produced by tumor-associated macrophages in B16F10 melanomas. Anticancer Res (2017) 37(7):3461-71. doi:10.21873/anticanres.11714

23. Wang H, Liang X, Li M, Tao X, Tai S, Fan Z, et al. Chemokine (CC motif) ligand 18 upregulates Slug expression to promote stem-cell like features by activating the mammalian target of rapamycin pathway in oral squamous cell carcinoma. Cancer Sci (2017) 108(8):1584-93. doi:10.1111/cas.13289

24. Antsiferova M, Piwko-Czuchra A, Cangkrama M, Wietecha M, Sahin D, Birkner K, et al. Activin promotes skin carcinogenesis by attraction and reprogramming of macrophages. EMBO Mol Med (2017) 9(1):27-45. doi:10.15252/ emmm.201606493

25. Wu X, Schulte BC, Zhou Y, Haribhai D, Mackinnon AC, Plaza JA, et al. Depletion of M2-like tumor-associated macrophages delays cutaneous T-cell lymphoma development in vivo. J Invest Dermatol (2014) 134(11):2814-22. doi:10.1038/jid.2014.206

26. König S, Nitzki F, Uhmann A, Dittmann K, Theiss-Suennemann J, Herrmann M, et al. Depletion of cutaneous macrophages and dendritic cells promotes growth of basal cell carcinoma in mice. PLoS One (2014) 9(4):e93555. doi:10.1371/ journal.pone. 0093555

27. Sugaya M, Miyagaki T, Ohmatsu H, Suga H, Kai H, Kamata M, et al. Association of the numbers of CD163(+) cells in lesional skin and serum levels of soluble CD163 with disease progression of cutaneous $\mathrm{T}$ cell lymphoma. J Dermatol Sci (2012) 68(1):45-51. doi:10.1016/j.jdermsci.2012.07.007

28. Kim YH, Tavallaee M, Sundram U, Salva KA, Wood GS, Li S, et al. Phase II investigator-initiated study of brentuximab vedotin in mycosis fungoides and Sézary syndrome with variable CD30 expression level: a multi-institution collaborative project. J Clin Oncol (2015) 33(32):3750-8. doi:10.1200/ JCO.2014.60.3969

29. Kakizaki A, Fujimura T, Kambayashi Y, Furudate S, Aiba S. Comparison of $\mathrm{CD} 163^{+}$macrophages and CD206 ${ }^{+}$cells in the lesional skin of CD30 lymphoproliferative disorders: lymphomatoid papulosis and primary cutaneous anaplastic large-cell lymphoma. Acta Derm Venereol (2015) 95(5):600-2. doi:10.2340/00015555-2016

30. Günther C, Zimmermann N, Berndt N, Grosser M, Stein A, Koch A, et al. Up-regulation of the chemokine CCL18 by macrophages is a potential immunomodulatory pathway in cutaneous T-cell lymphoma. Am J Pathol (2011) 179(3):1434-42. doi:10.1016/j.ajpath.2011.05.040

31. Getts DR, Turley DM, Smith CE, Harp CT, McCarthy D, Feeney EM, et al. Tolerance induced by apoptotic antigen-coupled leukocytes is induced by PD-L1+ and IL-10-producing splenic macrophages and maintained by T regulatory cells. J Immunol (2011) 7(5):2405-17. doi:10.4049/jimmunol.1004175

32. Linde N, Lederle W, Depner S, van Rooijen N, Gutschalk CM, Mueller MM. Vascular endothelial growth factor-induced skin carcinogenesis depends on recruitment and alternative activation of macrophages. J Pathol (2012) 227(1):17-28. doi:10.1002/path.3989

33. Rodriguez PC, Quiceno DG, Ochoa AC. L-arginine availability regulates T-lymphocyte cell-cycle progression. Blood (2007) 109(4):1568-73. doi:10.1182/ blood-2006-06-031856

34. Cyrus N, Mai-Anh Bui C, Yao X, Kohn LL, Galan A, Rhebergen AM, et al. Density and polarization states of tumor-associated macrophages in human cutaneous squamous cell carcinomas arising in solid organ transplant recipients. Dermatol Surg (2016) 42(Suppl 1):S18-23. doi:10.1097/ DSS.0000000000000371

35. Pico de Coaña Y, Poschke I, Gentilcore G, Mao Y, Nyström M, Hansson J, et al. Ipilimumab treatment results in an early decrease in the frequency of circulating granulocytic myeloid-derived suppressor cells as well as their Arginasel production. Cancer Immunol Res (2013) 1(3):158-62. doi:10.1158/ 2326-6066 
36. Werchau S, Toberer F, Enk A, Dammann R, Helmbold P. Merkel cell carcinoma induces lymphatic microvessel formation. J Am Acad Dermatol (2012) 67(2):215-25. doi:10.1016/j.jaad.2011.09.002

37. Kambayashi Y, Fujimura T, Furudate S, Hashimoto A, Haga T, Aiba S. Comparison of immunosuppressive cells and cytotoxic cells in angiosarcoma: the development of a possible supportive therapy for angiosarcoma. Dermatology (2013) 227(1):14-20. doi:10.1159/000351316

38. Zhu X, Yang J, Gao Y, Wu C, Yi L, Li G, et al. The dual effects of a novel peptibody on angiogenesis inhibition and M2 macrophage polarization on sarcoma. Cancer Lett (2017) 416:1-10. doi:10.1016/j.canlet.2017.10.043

39. Yamada K, Uchiyama A, Uehara A, Perera B, Ogino S, Yokoyama Y, et al. 6-MFG-E8 drives melanoma growth by stimulating mesenchymal stromal cell-induced angiogenesis and M2 polarization of tumor-associated macrophages. Cancer Res (2016) 76(14):4283-92. doi:10.1158/0008-5472. CAN-15-2812

40. Heissig B, Hattori K, Dias S, Friedrich M, Ferris B, Hackett NR, et al. Recruitment of stem and progenitor cells from the bone marrow niche requires MMP-9 mediated release of kit-ligand. Cell (2002) 109(5):625-37. doi:10.1016/S0092-8674(02)00754-7

41. Kale S, Raja R, Thorat D, Soundararajan G, Patil TV, Kundu GC. Osteopontin signaling upregulates cyclooxygenase-2 expression in tumor-associated macrophages leading to enhanced angiogenesis and melanoma growth via $\alpha 9 \beta 1$ integrin. Oncogene (2014) 33(42):2295-306. doi:10.1038/onc. 2015.315

42. Litvinov IV, Netchiporouk E, Cordeiro B, Doré MA, Moreau L, Pehr K, et al. The use of transcriptional profiling to improve personalized diagnosis and management of cutaneous T-cell lymphoma (CTCL). Clin Cancer Res (2015) 21(12):2820-9. doi:10.1158/1078-0432.CCR-14-3322

43. Mantovani A, Allavena P, Sica A, Balkwill F. Cancer-related inflammation. Nature (2008) 454(7203):436-44. doi:10.1038/nature07205

44. Rogers TL, Holen I. Tumour macrophages as potential targets of bisphosphonates. J Transl Med (2011) 9:177. doi:10.1186/1479-5876-9-177

45. Melani C, Sangaletti S, Barazzetta FM, Werb Z, Colombo MP. Aminobiphosphonate-mediated MMP-9 inhibition breaks the tumor-bone marrow axis responsible for myeloid derived suppressor cell expansion and macrophage infiltration in tumor stroma. Cancer Res (2007) 67(23):11438-46. doi:10.1158/0008-5472.CAN-07-1882

46. Mitchem JB, Brennan DJ, Knolhoff BL, Belt BA, Zhu Y, Sanford DE, et al. Targeting tumor-infiltrating macrophages decreases tumor-initiating cells, relieves immunosuppression, and improves chemotherapeutic responses. Cancer Res (2013) 73(3):1128-41. doi:10.1158/0008-5472.CAN-12-2731

47. Sevko A, Michels T, Vrohlings M, Umansky L, Beckhove P, Kato M, et al. Antitumor effect of Paclitaxel is mediated by inhibition of myeloid-derived suppressor cells and chronic inflammation in the spontaneous melanoma model. J Immunol (2013) 190(5):2464-71. doi:10.4049/jimmunol.1202781

48. Bruchard M, Mignot G, Derangère V, Chalmin F, Chevriaux A, Végran F, et al. Chemotherapy-triggered cathepsin B release in myeloid-derived suppressor cells activates the Nlrp3 inflammasome and promotes tumor growth. Nat Med (2013) 19(1):57-64. doi:10.1038/nm.2999

49. Fujimura T, Takahashi K, Kambayashi Y, Furudate S, Hidaka T, Kakizaki A, et al. Retrospective study of cutaneous squamous cell carcinoma on the lip treated with peplomycin administered through a superficial temporal artery. Anticancer Res (2017) 37(4):1885-9. doi:10.21873/anticanres.11526

50. Fujimura T, Kambayashi Y, Furudate S, Kakizaki A, Haga T, Hashimoto A, et al. Immunomodulatory effects of peplomycin on immunosuppressive and cytotoxic cells in the lesional skin of cutaneous squamous cell carcinoma. Dermatology (2015) 230(3):250-5. doi:10.1159/000369166

51. Georgoudaki AM, Prokopec KE, Boura VF, Hellqvist E, Sohn S, Östling J, et al. Reprogramming tumor-associated macrophages by antibody targeting inhibits cancer progression and metastasis. Cell Rep (2016) 15(9):2000-11. doi:10.1016/j.celrep.2016.04.084
52. Gordon SR, Maute RL, Dulken BW, Hutter G, George BM, McCracken MN, et al. PD-1 expression by tumour-associated macrophages inhibits phagocytosis and tumour immunity. Nature (2017) 545(7655):495-9. doi:10.1038/ nature 22396

53. Vilain RE, Menzies AM, Wilmott JS, Kakavand H, Madore J, Guminski A, et al. Dynamic changes in PD-L1 expression and immune infiltrates early during treatment predict response to PD-1 blockade in melanoma. Clin Cancer Res (2017) 23(17):5024-33. doi:10.1158/1078-0432.CCR-16-0698

54. Young HL, Rowling EJ, Bugatti M, Giurisato E, Luheshi N, Arozarena I, et al. An adaptive signaling network in melanoma inflammatory niches confers tolerance to MAPK signaling inhibition. J Exp Med (2017) 214(6):1691-710. doi:10.1084/jem.20160855

55. Kumar V, Donthireddy L, Marvel D, Condamine T, Wang F, Lavilla-Alonso S, et al. Cancer-associated fibroblasts neutralize the anti-tumor effect of CSF1 receptor blockade by inducing PMN-MDSC infiltration of tumors. Cancer Cell (2017) 32(5):654.e-68.e. doi:10.1016/j.ccell.2017.10.005

56. Lyons YA, Pradeep S, Wu SY, Haemmerle M, Hansen JM, Wagner MJ, et al. Macrophage depletion through colony stimulating factor 1 receptor pathway blockade overcomes adaptive resistance to anti-VEGF therapy. Oncotarget (2017) 8(57):96496-505. doi:10.18632/oncotarget.20410

57. Pradel LP, Ooi CH, Romagnoli S, Cannarile MA, Sade H, Rüttinger D, et al. Macrophage susceptibility to emactuzumab (RG7155) treatment. Mol Cancer Ther (2016) 15(12):3077-86. doi:10.1158/1535-7163.MCT-16-0157

58. Miyagaki T, Sugaya M, Suga H, Ohmatsu H, Fujita H, Asano Y, et al. Increased CCL18 expression in patients with cutaneous T-cell lymphoma: association with disease severity and prognosis. JEur Acad Dermatol Venereol (2013) 27(1):e60-7. doi:10.1111/j.1468-3083.2012.04495.x

59. Zhang Q, Qin J, Zhong L, Gong L, Zhang B, Zhang Y, et al. CCL5-mediated Th2 immune polarization promotes metastasis in luminal breast cancer. Cancer Res (2015) 75(20):4312-21. doi:10.1158/0008-5472.CAN-14-3590

60. Ding H, Zhao L, Dai S, Li L, Wang F, Shan B. CCL5 secreted by tumor associated macrophages may be a new target in treatment of gastric cancer. Biomed Pharmacother (2016) 77:142-9. doi:10.1016/j.biopha.2015.12.004

61. Van Gorp H, Delputte PL, Nauwynck HJ. Scavenger receptor CD163, a Jack-of-all-trades and potential target for cell-directed therapy. Mol Immunol (2010) 47(7-8):1650-1460. doi:10.1016/j.molimm.2010.02.008

62. Fujimura M, Fujimura T, Kakizaki A, Sato-Maeda M, Niizuma K, Tomata Y, et al. Increased serum production of soluble CD163 and CXCL5 in patients with moyamoya disease: possible involvement of autoimmunity in its pathogenesis. Brain Res (2017) 1679:39-44. doi:10.1016/j.brainres.2017.11.013

63. Fujimura T, Kakizaki A, Furudate S, Aiba S. A possible interaction between periostin and $\mathrm{CD} 163+$ skin-resident macrophages in pemphigus vulgaris and bullous pemphigoid. Exp Dermatol (2016) 26(12):1193-8. doi:10.1111/ exd.13157

64. Fujimura T, Kambayashi Y, Furudate S, Kakizaki A, Hidaka T, Haga T, et al. Isolated ACTH deficiency possibly caused by nivolumab in a metastatic melanoma patient. J Dermatol (2017) 44(3):e13-4. doi:10.1111/1346-8138.13532

65. Fujimura T, Hidaka T, Kambayashi Y, Furudate S, Kakizaki A, Tono H, et al. Phase I study of nivolumab combined with IFN- $\beta$ for patients with advanced melanoma. Oncotarget (2017) 8(41):71181-7. doi:10.18632/oncotarget.17090

Conflict of Interest Statement: The authors declare that the research was conducted in the absence of any commercial or financial relationships that could be construed as a potential conflict of interest.

Copyright (c) 2018 Fujimura, Kambayashi, Fujisawa, Hidaka and Aiba. This is an open-access article distributed under the terms of the Creative Commons Attribution License (CC BY). The use, distribution or reproduction in other forums is permitted, provided the original author $(s)$ or licensor are credited and that the original publication in this journal is cited, in accordance with accepted academic practice. No use, distribution or reproduction is permitted which does not comply with these terms. 